\title{
Teaching Sensor Fusion and Kalman Filtering using a Smartphone
}

\author{
Gustaf Hendeby, Fredrik Gustafsson and Niklas Wahlström
}

Linköping University Post Print

Tweet

N.B.: When citing this work, cite the original article.

Original Publication:

Gustaf Hendeby, Fredrik Gustafsson and Niklas Wahlström, Teaching Sensor Fusion and Kalman Filtering using a Smartphone, 2014, Proceedings of the 19th World Congress of the International Federation of Automatic Control (IFAC).

Postprint available at: Linköping University Electronic Press

http://urn.kb.se/resolve?urn=urn:nbn:se:liu:diva-109947 


\title{
Teaching Sensor Fusion and Kalman Filtering using a Smartphone ${ }^{\star}$
}

\author{
Gustaf Hendeby ${ }^{* \dagger}$ Fredrik Gustafsson * Niklas Wahlström* \\ * Dept. of Electrical Engineering \\ Linköping University, Sweden \\ e-mail: \{hendeby, fredrik, nikwa\}@isy.liu.se \\ ${ }^{\dagger}$ Div. of Sensor Informatics \\ Dept. of Sensor \& EW Systems \\ Swedish Defence Research Agency \\ Linköping, Sweden
}

\begin{abstract}
The Kalman filter has been the work horse in model based filtering for five decades, and basic knowledge and understanding of it is an important part of the curriculum in many Master of Science programs. It is therefore important to combine theoretical studies with practical experience to allow the students to deepen their understanding of the filter. We have developed a lab where the students implement a Kalman filter in a real-time MATLAB framework, to which data are streamed from the smartphone over WiFi. The goal of the lab is to estimate the orientation of the smartphone, which can be nicely visualized graphically and also be compared to the built-in filters in the smartphone. The filter can accept any combination of sensor data from accelerometers, gyroscopes, and magnetometer, with different performance. Different tunings and tricks in the Kalman filter are easily evaluated on-line. The smartphone app is also a stand-alone tool to visualize the sensor data graphically. So far the lab seems to have been successful in reaching the pedagogic goals and to engage the students.
\end{abstract}

Keywords: Kalman filtering; Teaching sensor fusion; Student lab; Smartphone; Orientation estimation

\section{INTRODUCTION}

Navigation is one of the first and one of the most important applications of the Kalman filter, and a good example of sensor fusion, where several complementary sensors are needed to solve a nontrivial problem. At the same time, it is a quite challenging application from a complexity and a numerical point of view. A core component of any navigation system is the orientation filter that integrates inertial information from gyroscopes and accelerometers, with magnetometer measurements and other supporting sensors that relate to the orientation of the platform with respect to the world. Orientations are furthermore very concrete and intuitive to understand, making it easy to illustrate properties of the estimate.

The usage of smartphones has seen a huge increase the last couple of years. Today, most people carry around a competent mobile computer designed for interaction with the environment. These smartphones are fitted with inertial sensors, GPS, light and proximity sensors, as well as microphones and cameras. The radio receivers measure signal strength from various wireless networks. The topof-the-line models host even more and better sensors. This makes these devices highly interesting from a sensor fusion point of view, providing for multi-modal sensory information and computing power in small easily accessible package (Lane et al., 2010; Ma et al., 2013).

Altogether, this makes designing a lab around developing an orientation filter for smartphone data a good idea;

\footnotetext{
* This work was supported by the SSF funded project Cooperative Localization.
}

offering both the possibility to give theoretical insights and practical experience of sensor fusion and filter implementation in particular. Using data streamed in real time from a smartphone allows the students to gain practical experience from filter design, filter tuning, disturbance rejection, and sensitivity issues. Using a smartphone as sensor platform and distributing the necessary software for data collection for free, furthermore, encourages the students to continue exploring their own smartphones outside the lab.

This paper describes a lab used in the graduate course Sensor Fusion at Linköping University (TSRT14, 2013), and the experiences made developing and teaching it. In Section 2 the course goals are described and Section 3 discusses related literature. In Section 4, the lab and the theory that it intends to teach and the practical experiences it should give are described. Section 5 presents the developed Android app and the necessary infrastructure. Section 6 further discusses the design of the lab and how it helps to reach the goals set up for it, and offers lessons learned from the first time it was given. Conclusions, as far as possible, are drawn in Section 7.

\section{COURSE GOALS}

The Kalman filter theory is mainly taught in two courses at Linköping University:

- The course Digital Signal Processing (TSRT78, 2013) teaches the linear discrete time Kalman filter using the textbook (Gustafsson et al., 2010). 
- The course Sensor Fusion (TSRT14, 2013) treats the Kalman filter from a sensor fusion perspective, as well as describes various variants of nonlinear filters. It is based on the book (Gustafsson, 2010).

This section describes the goals of a lab in the latter course.

The course curriculum for the Sensor Fusion course at Linköping University states the following pedagogical goals (LiTH, 2013; TSRT14, 2013):

[...] after the course the student should have the ability to:

(i) understand the fundamental principles in estimation and detection theory;

(ii) implement algorithms for parameter estimation in linear and nonlinear models;

(iii) implement algorithms for detection and estimation of the position of a target in a sensor network;

(iv) apply the Kalman filter to linear state-space models with a multitude of sensors;

(v) apply nonlinear filters (extended Kalman filter (EKF), unscented Kalman filter (UKF), particle filter (PF)) to non-linear or non-Gaussian state-space models;

(vi) implement basic algorithms for simultaneous localization and mapping (SLAM);

(vii) describe and model the most common sensors used in sensor fusion applications;

(viii) implement the most common motion models in target tracking and navigation applications; and

(ix) understand the interplay of the above in a few concrete real applications.

Several means can be used to reach these goals: lectures, exercises sessions, and lab sessions. Lectures are a good way to introduce theory, whereas tutorial sessions can train the students in working with selected algorithms. Labs are unique in that they also provide firsthand experience of methods and actual practical problems usually ignored in most textbooks in favor of expressing the theory more clearly. With this in mind, the lab described here intend to contribute to the fulfillment of the following the pedagogic goals: (i), (v), (vii), and (ix). The lab is complementary to the textbook (Gustafsson, 2010) presentation, and the main focus of the lab is to give the students practical experience of an actual estimation problem.

\section{RELATED LITERATURE}

Kirschner and Meester (1988) have identified four major premises for conducting successful laboratory work in the teaching of natural sciences. The first premise is illustration and concretization which allows the students to grasp the complex nature of science by the use of concrete material and the possibility to manipulate. The second premise deals with cognitive aspects which emphasizes the student participation in the laboratory experience, such as collection of data and analysis of real phenomena. The third premise states the importance of practical skills, whereas the fourth premise deals with the students' motivation and the importance that they enjoy the activities and the practical work.

When it comes to engineering laboratory work, Feisel and Rosa (2005) among others have noticed a lack of coherent learning objectives in the literature. They claim that many educators have not defined the objectives at all or have done so in terms which are difficult to check if they are fulfilled or not. Therefore Feisel and Rosa have listed thirteen objectives originating from a meeting including some fifty distinguished engineering educators discussion the question "What are the fundamental objectives of engineering instructional laboratories?". The first six of these objectives deals with the practical skills and include that the student shall be able to (1) apply appropriate sensors and software tools to make measurements, (2) identify strength and limitations of theoretical models of realworld behaviors, (3) devise an experimental approach, (4) demonstrate the ability to collect, analyze and interpret data, (5) designing, testing and debugging a system, and (6) learn from failure by identifying unsuccessful outcomes and then re-engineer effective solutions.

In this context it is also important to more precisely define the educational benefits for the students to collect their own experimental data. Ma and Nickerson (2006) have looked into the effectiveness of the laboratories for fulfilling learning objectives where they have performed a literature survey by comparing hands-on versus simulated and remote labs. According to their findings, hands-on labs do have benefits, primarily for training the students in their design skills. However, when it comes to conceptual understanding and professional skills no major benefit of hands-on labs could be found. Feisel and Rosa (2005) also distinguish between different types of labs, development, research, and educational labs, depending on their purposes. The development lab has most similarities with how engineers practice their profession. Development engineers need to collect experimental data for designing and developing a product and also to determine if the product fulfills the requirements and to analyze if changes have to be made.

The motivation for and the joy doing the laboratory work is also important in order to improve the attitude and interest for science. This was articulated by Shulman and Tamir (1973) who claimed that the attitude and imagination are as important outcomes from scientific teaching as their cognitive counterparts.

It is also easy to understand that motivation and enjoying the task has a positive effect on the learning. In science education, an interesting laboratory work is important, which is also emphasized in the science education literature. Hofstein and Lunetta (2004) state

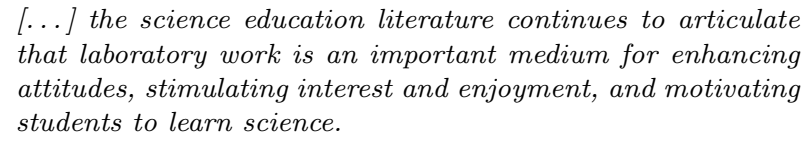

Hofstein and Lunetta (2004) also discuss the benefits of incorporating appropriate high technology tools in the laboratory work to enable the students to make more interesting investigations. They claim

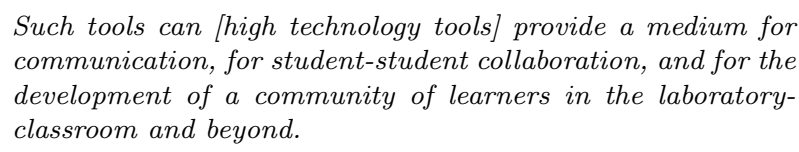

\section{LAB EXERCISE}

In this section the underlying estimation problem used in the lab is described in more detail. Based on this description it is outlined which theoretical aspects and 


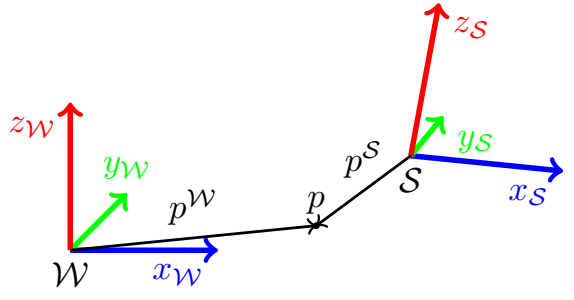

Fig. 1. Illustration of the two involved inertial frames; the world frame, $\mathcal{W}$, and the sensor (smartphone) fixed frame, $\mathcal{S}$.

practical considerations the students face during the lab. This is done to substantiate what the lab is intended to teach.

\subsection{Estimation Problem}

Orientation estimation is a common task that is also easily visualized and experienced using the sensors in a smartphone. Furthermore, modern smartphones also have built-in algorithms to provide their own orientation estimate. This estimate can be used by students as a reasonable ground truth to compare and compete with. The basic steps of estimating the orientation are described below.

The smartphone's local coordinate system, $\mathcal{S}$, relates to the global coordinate system, $\mathcal{W}$, via the affine transformation

$$
p^{\mathcal{W}}=R^{\mathcal{W} / \mathcal{S}} p^{\mathcal{S}}+t^{\mathcal{W} / \mathcal{S}}
$$

that describes the coordinate of a point, $p$, in $\mathcal{W}$ as a function of the coordinate in $\mathcal{S}$, as illustrated in Fig. 1. The orientation of the smartphone is defined by the rotation $R^{\mathcal{W} / \mathcal{S}}$, whereas the displacement of the device is given by $t^{\mathcal{W} / \mathcal{S}}$. The displacement $t^{\mathcal{W} / \mathcal{S}}$ cannot reliably be estimated with inertial and magnetic measurements alone (without resorting to tricks and making more assumptions); hence, the objective of the lab is limited to estimating the sensor rotation $R^{\mathcal{W} / \mathcal{S}}$.

In the lab, three kinds of measurements are used to solve the orientation estimation task, described in turn below.

First, accelerometers measure body accelerations, expressed in the senor frame, $\mathcal{S}$,

$$
y^{a}=\left(R^{\mathcal{S} / \mathcal{W}}\right)^{T} g^{0}+F+e^{a},
$$

where $g^{0}$ is the nominal gravity vector expressed in $\mathcal{W}$, $F$ the specific force acting on the device, and $e^{a}$ the measurement noise. Assuming negligible movements of the smartphone and only aiming to extract the orientation, the specific force, $F$, is often ignored, i.e., $F \approx 0$. Measuring the gravity provides information to properly align the horizontal plane, but cannot help defining a forward direction. The implications of this common approximation are illustrated in the lab.

The magnetic field has a component in the horizontal plane, and can hence define a forward direction. The magnetometer provides measurements of the magnetic field in $\mathcal{S}$,

$$
y^{m}=\left(R^{\mathcal{S} / \mathcal{W}}\right)^{T} m^{0}+e^{m},
$$

where $m^{0}$ is the nominal magnetic field in $\mathcal{W}$, and $e^{m}$ is measurement noise. In practice the magnetic field is often heavily disturbed, especially in indoor environments, which raises important questions about the best way to use magnetic measurements and minimize the implications of disturbances.

The last measurement used is the angular velocities measured with the gyroscopes. The angular velocities are measured in $\mathcal{S}$. The measurements can either be interpreted as normal measurements and treated analogously to the acceleration and magnetic field, or be considered measured inputs to be integrated to obtain an approximate orientation,

$$
\begin{aligned}
q_{k+1} & =e^{\frac{1}{2} S\left(\omega_{k}+w_{k}\right) T} q_{k} \\
& =\cos \left(\frac{T\left\|\bar{\omega}_{k}\right\|}{2}\right) I q_{k}+\frac{T}{2} \operatorname{sinc}\left(\frac{T \| \bar{\omega}_{k} \mid}{2}\right) S\left(\bar{\omega}_{k}\right) q_{k} \\
& \approx\left(I+\frac{T}{2} S\left(\omega_{k}\right)\right) q_{k}+\frac{T}{2} \bar{S}\left(q_{k}\right) w_{k} .
\end{aligned}
$$

In this description a unit quaternion, $q_{k}$, is used to efficiently represent the rotation $R^{\mathcal{S}} / \mathcal{W}$ at time $k$, and $\bar{\omega}_{k}=\omega_{k}+w_{k}$, where $\omega_{k}$ are the measured angular rates and $w_{k}$ process noise (mainly consisting of measurement noise). Furthermore, $S$ and $\bar{S}$ are skew-symmetric matrix representations of the cross-product operation from left and right, respectively.

\subsection{Covered Theoretical Aspects}

The orientation estimation problem, as briefly described above, offers the possibility to deal with several important theoretical aspects of sensor fusion. When approaching the orientation estimation problem as a filtering problem, the students encounter both nonlinear dynamic and nonlinear measurement equations. Hence, they are forced to apply a nonlinear filter to solve the problem; in this case implementing an extended Kalman filter (EKF). In the process, they must understand how accelerometers, magnetometers, and gyroscope work in order to implement the appropriate dynamic equation (4b) as well as the measurement equations (2) and (3).

The problem also opens up for interesting discussions regarding how to represent rotations. Using a rotation matrix would offer the most familiar representation for most students. However, the matrix representation is heavily overparametrized, and a poor alternative for the task. Instead the lab introduces quaternions for the filter constructed in the lab. This way the students get hands on experience of working with this very common representation of objects in the $\mathbb{S} \mathbb{O}^{3}$ group.

Another important discussion is how the available measurements should be utilized. For instance, what is the difference between using the measurements of angular velocities from the gyroscope as inputs to the dynamic equation or as measurements, after augmenting the state with angular velocities, and how many biases need to be and can be estimated.

\subsection{Covered Practical Aspects}

The theoretical side of the lab is naturally complemented by a wide range of practical experience as a consequence of working with data from commercial sensors in real time. In the lab, the students are given a MATLAB script to extract data from a smartphone in real-time. Based on this and 


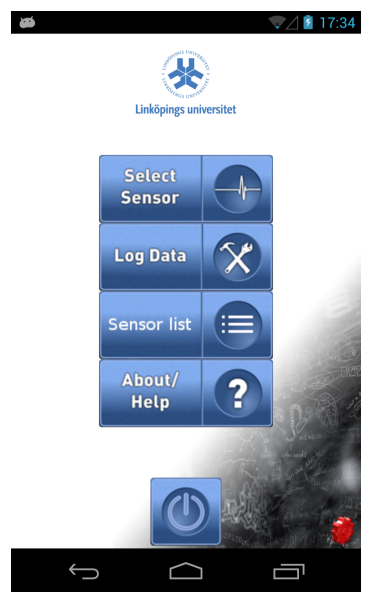

(a) The app main view.

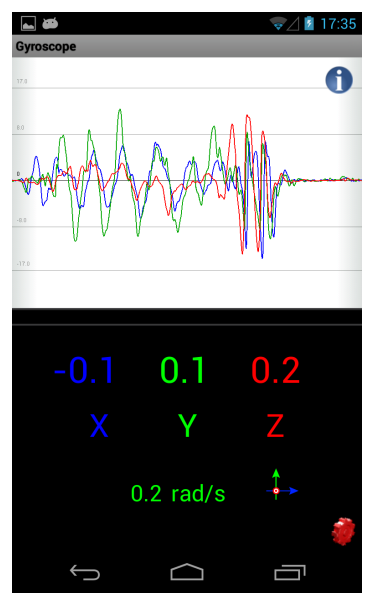

(b) Live graph of sensor data.

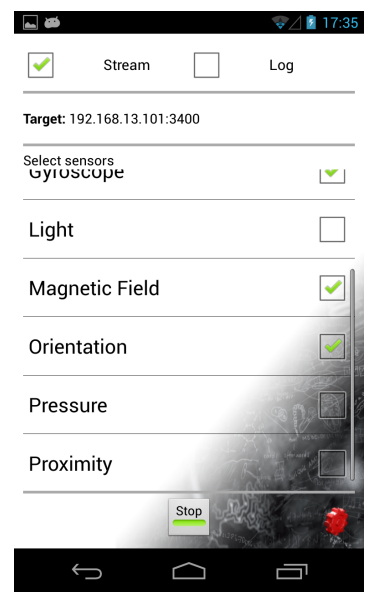

(c) Log and streaming view.

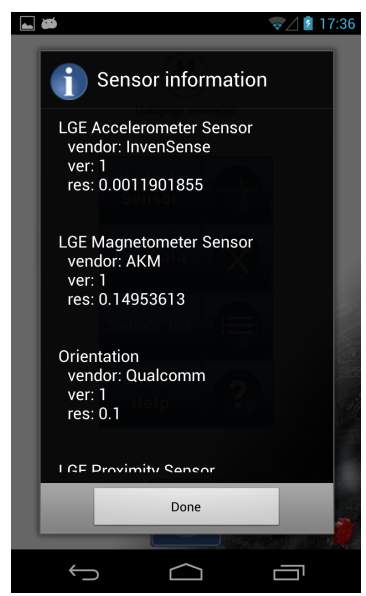

(d) Sensor information dia$\log$.
Fig. 2. Views from the Sensor Fusion app.

the descriptions in the lab instructions, an orientation filter should then be constructed. In the process, the students are given ample opportunity to use their filtering skills on a real application.

In order for the lab to have the expected effect, it is important that the students spend their time doing sensor fusion rather than trying to understand a complex framework in which to perform the task. For this reason, it has been important to implement the lab framework in such a way that the students can work completely in MATLAB, an environment they are familiar with from other courses.

In MATLAB the students are asked to implement the different steps in the orientation filter. Doing this they get practical experience from implementing the nonlinear EKF and for this how to linearize measurement equations. The results can directly be compared to orientation estimates available in the phone.

A fundamental component for successful signal processing is to understand the available signals and the studied system. Therefore, the first thing the students are asked to do is to get acquainted to the sensors and how they behave, and then to design simple calibration experiments. The students analyze the results and identify biases, potential drifts, and other peculiarities of the sensors. Different devices suffer from different problems, which makes the exercise extra interesting. Given their findings, the students should compensate their measurements. In practice this boils down to compensating for gyroscope bias. The calibration experiment is also used to get a good initial tuning for the filter.

The need for outlier rejection is easily illustrated by asking the students to shake their smartphone and/or to introduce magnetic disturbances. Not only does the students' textbook estimate fail, it is also easy to observe that the orientation estimate provided by the internal software in the smartphone automatically compensates for these effects. The students then implement their own outlier rejection and can then aim to outperform the builtin algorithm. Properly done, surprisingly good results can be achieved in short time.
Most important is the experiences the students gain in dealing with practical signal processing, realizing the difference between the theory and practice. Hopefully, the lab makes the students much more aware of the differences between textbook examples taught in the lectures and practical problems.

\section{SENSOR FUSION APP}

The Sensor Fusion app used in the described lab has been developed at Linköping University as an Android based teaching and demonstration platform for sensor fusion. The app is available for free under the name Sensor Fusion $a p p^{1}$ from Google Play Store for everyone with a device running Android v. 2.3.3 (Gingerbread) or later. This section describes the app; what it can do and how it can be used to create a real-time connection between the smartphone and, e.g., MATLAB running on standard desktop computer. Currently, students without an Android device and those who do not want to use their own smartphone, are provided relatively cheap Google Nexus 4 smartphones ${ }^{2}$ to use during the lab. The sensors in the Nexus 4 are evaluated in (Ma et al., 2013).

\subsection{App Description}

When starting up the Sensor Fusion app, the main menu appears as seen in Fig. 2(a). The menu offers the user the main functionality available:

- real-time visualization of sensor measurement, using Select Sensor;

- logging or streaming measurements, using Log Data; and

- getting information about what sensors are available in the current device, using Sensor List. (See Fig 2(d).)

\footnotetext{
1 The Sensor Fusion app at Google Play Store: http://goo.gl/OqNyU

2 Google Nexus 4 on Wikipedia:

http://en.wikipedia.org/wiki/Nexus_4/

Google has at the time of writing this discontinued the Google Nexus 4 in favor of the Google Nexus 5 phone.
} 


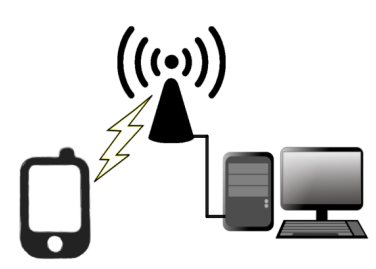

Fig. 3. The setup used to connect the smartphones to the computers in the lab during the lab session. The smartphone, to the left, is connected via $\mathrm{WiFi}$ to a wireless access point, that in its turn is connected to the computer, shown to the right, via a wired network.

The app also offers some options to configure its behavior.

The real-time visualization of sensor data works for all the main sensors available in modern Android device. Fig. 2(b) shows how gyroscope measurements are visualized. Each sensor view furthermore offers a dialog explaining what physical quantity the sensor measures, how it can be used, and suggests easy experiments to perform to gain a better understanding of the measurements. The ability to visualize measurements from the sensors direct on the smartphone is used in the initial stage of the lab to help the students get to know the sensors they use in the lab.

From the perspective of the lab, the streaming and logging capability of the app is the most important. From one unified view, see Fig. 2(c), it is possible to in real time stream selected sensors to a server and/or log the measurements to a log file on the device for off-line analysis. The streaming functionality is vital to the lab experience, and is therefore described in more detail below.

\subsection{Streaming Measurements}

The Sensor Fusion app streams data by opening a TCP connection to a minimal server program running on the receiving end, e.g., MATLAB on a laptop or a lab computer. The app is fully configurable with regard to which IP address to stream data to and which port to use. The app can hence make use of any available WiFi and mobile Internet connection to stream the data. This also means it will automatically make use of VPN connections if present. This allows for easy use in almost any setting. It also makes it possible to use either a laptop or the device itself as a wireless hotspot to connect the two to obtain a truly mobile system not relying on any external infrastructure. In the labs, the students connect the phones to the local wireless network to stream the data. The lab setup is illustrated in Fig. 3 utilizing the normal WiFi infrastructure in the computer labs.

The computer receiving the streamed measurement data should run a small server. For this purpose, a small Java library is provided that can be downloaded via a link on Google Play Store. The Java library is written in such a way that it can be easily embedded in MATLAB or used stand alone as a part of a Java program that utilizes the streamed measurements. The format used to stream the data furthermore allows for quite easily developing servers in different languages if needed.

The MATLAB based server allows for full integration of the streamed measurements into MATLAB. At the beginning of

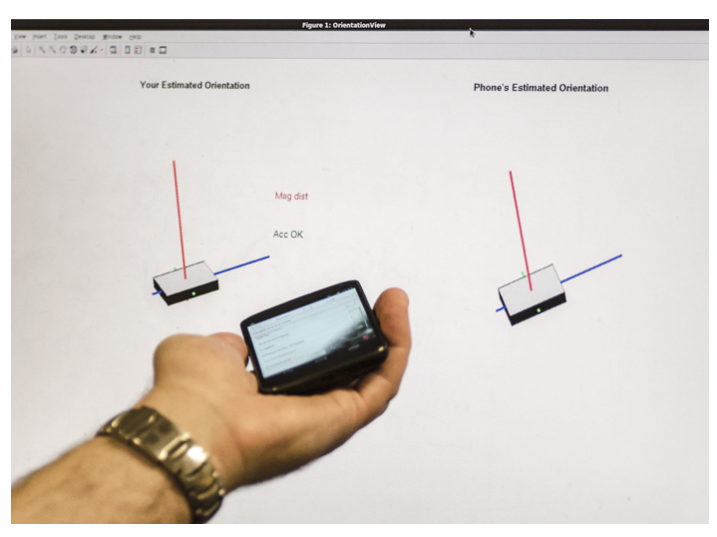

Fig. 4. Student evaluating his orientation estimate comparing the phone orientation with the estimated orientation on the screen on his own laptop.

the lab a skeleton of MATLAB code is provided, and the students then extends it during the lab. The code shows how to access the streamed data, and provides an easy way to visualize the orientations they estimate (see Fig. 4). The code skeleton is also available following the links on the app's Google Play Store page. By providing easy access to measurement data in this way allows students with limited coding experience to focus on the sensor fusion aspects of the lab, rather than on how to obtain data from the device.

\section{DISCUSSION AND LESSONS LEARNED}

At the time this paper is written, the lab and the Sensor Fusion app has been used in the Senor Fusion course once. The overall experiences are positive, but due to the limited experiences, it is too early to make any farreaching conclusions. Hence, this section discusses ideas and experiences from the preparations, execution, and post-processing of the lab, and tries to relate this to available literature. Hopefully, this description can inspire others to construct useful labs for teaching sensor fusion.

In order to motivate the usage of a lab in a course, it should in a clear way help to fulfill the pedagogic goals of the course. In this case the pedagogic goals of the course is listed in Sec. 2. These goals will be referred to as (i) $-(i x)$ below.

The described lab contributes to (i) (understanding fundamental principles of estimation theory) in that it allows and encourages the students to play around with a nontrivial estimation problem. During the lab the students experience how using (or not using) different signals affects the end result, as well as the effects of sensor bias, disturbances and outliers. Hence, the students are forced to think about how to use the signals available and if they should estimate biases or not. In order to do this they need to understand the underlying estimation principles.

The filtering problem requires a nonlinear filter (an EKF in this case) to be solved. This works toward $(v)$ in the course. In the process, the students have to design and implement measurement models for three common sensors (accelerometer, gyroscope, and magnetometer). They furthermore experience problems, such as environmental disturbances and outliers, characteristic to these sensor types. This is part of the fulfillment of (vii). 
The lab is designed with the practical learning objectives for engineering labs, as formulated by Feisel and Rosa (2005), in mind. The students are part of the whole signal processing chain; collecting data from the smartphone, evaluating the data available and based on that, design the filter needed for estimating the orientation. Finally, they are then guided to put the solution in place. In the process learning from mistakes and experiences they make. The lab instructions are intentionally written in such a way that the students try simple things first, and are then forced to identify the shortcomings with these simple solutions and come up with solutions to them and reengineer accordingly.

This practical hands-on approach to the lab is according to Ma and Nickerson (2006) a good way to prepare students for procedures common in the engineering profession. This relates closely to pedagogic goal (ix). The main objection to hands-on labs is usually the economic cost to maintain expensive equipment. The lab presented in this paper is fortunately relatively cheap in material and maintenance. Many students use their own smartphones for data collection, and the smartphones offered to those without appropriate smartphone, or to those who do not want to use their own phones, can be acquired at a very affordable price compared to other lab equipment.

During the lab sessions, some students indicated they had played around with the app and the measurements before coming to the lab. This is a good sign that the topic of lab and the easy access to the free supporting software inspired these students to deepen their knowledge. Furthermore, the lab has proved to be compatible with a wide selection of smartphones making it very accessible. This is further demonstrated by almost two thirds of the students using their own smartphone during the labs and the close to 1500 installations of the Sensor Fusion app.

It was also the experience during the lab that many students found the exercise engaging. They seemed positive to have a lab based around a smartphone, and enjoyed to see what they could do with the sensors many of them owned and carried around in their pocket each day. Hopefully, and as indicated by some of the students, they do not stop their laboratory work at the end of the time assigned for the lab. Contrary, hopefully the easy accessible smartphone to smartphone measurements invites to further experiments as suggested by Hofstein and Lunetta (2004). At the same time, the data collected in real-time allows them to experiment and analyze different solutions as they go on. This way the lab well complies with the four main principles for successful lab according to Kirschner and Meester (1988).

At the same time as using a smartphone for the lab seemed to engage most of the students, it also posed an initial barrier for students not acquainted to the technology. This is unfortunate, and shows how important clear instructions are and not to rely on students being familiar with the technology.

\section{CONCLUSIONS AND FURTHER DEVELOPMENT}

This paper describes a lab designed for the Sensor Fusion course at Linköping University. In the lab, the students estimate the orientation of a smartphone using measure- ments from the phone streamed in real-time from an app developed for the purpose. The app and the material for the lab are available for free. The initial experiences from the lab are positive and the students seem engaged.

The Sensor Fusion app can also serve as a demonstration platform for sensor fusion. The app can also be used to collect data for other purposes.

In the future, there are plans to extend the app so that it can collect an even wider range of data from the phone; such as signal strength from WiFi access points and other wireless cellular systems, as well as sound from the microphones and images from the built-in camera. This would allow for even more advanced usage of the app both in teaching and as a cheap sensor platform for conducting research.

\section{ACKNOWLEDGEMENTS}

We would like to acknowledge the work put into developing the first version of the Sensor Fusion app; it was developed in collaboration with $\mathrm{HiQ}$ (http://www.hiq.se), funded by a SAAB award in the name of former CEO Ảke Svensson received by Prof. Fredrik Gustafsson. We also want to thank all students and teaching assistants who made this lab run as smoothly as it did.

\section{REFERENCES}

L. D. Feisel and A. J. Rosa. The role of the laboratory in undergraduate engineering education. Journal of Engineering Education, 94(1):121-130, 2005.

F. Gustafsson. Statistical Sensor Fusion. Studentlitteratur $\mathrm{AB}, 2010$.

F. Gustafsson, L. Ljung, and M. Millnert. Signal Processing. Studentlitteratur AB, 2010.

A. Hofstein and V. N. Lunetta. The laboratory in science education: Foundations for the twenty-first century. Science education, 88(1):28-54, 2004.

P. A. Kirschner and M. A. M. Meester. The laboratory in higher science education: Problems, premises and objectives. Higher Education, 17(1):81-98, 1988.

N. D. Lane, E. Miluzzo, Hong Lu, D. Peebles, T. Choudhury, and A. T. Campbell. A survey of mobile phone sensing. IEEE Commun. Mag., 48(9):140-150, 2010.

LiTH, 2013. TSRT14 Sensor Fusion. Linköping Institute of Technology, 2013. http://kdb-5.liu.se/liu/ lith/studiehandboken/enkursplan. lasso?\&k_ kurskod=TSRT14\&k_budget_year=2013.

J. Ma and J. V. Nickerson. Hands-on, simulated, and remote laboratories: A comparative literature review. ACM Computing Surveys (CSUR), 38(3):7, 2006.

Z. Ma, Y. Qiao, B. Lee, and E. Fallon. Experimental evaluation of mobile phone sensors. In 24th IET Irish Signals and Systems Conference, Letterkenny, Ireland, June 2013.

L. D. Shulman and P. Tamir. Research on teaching the natural science. In R. M. W. Travers, editor, Second handbook of research on teaching, pages 1098-1140. Chicago: Rand McNally, 1973.

TSRT14, 2013. TSRT14 sensor fusion, 2013. URL http: // www. control.isy.liu.se/en/student/tsrt14/.

TSRT78, 2013. TSRT78 digital signal processing, 2013. URL http://www.control.isy.liu.se/en/student/ tsrt78/. 